\title{
Handlungsarena Metropolregionen
}

\section{Strategische Ansatzpunkte für Nachhaltigkeit in Stadtregionen}

\author{
Metropolregionen sind in Gestalt und \\ Zielsetzungen vielfältig. Ein Blick auf Entwick- \\ lungsmöglichkeiten jenseits der dominierenden \\ Wachstums- und Wettbewerbsorientierung \\ eröffnet dabei neue Perspektiven. In ausgewähl- \\ ten Handlungsfeldern könnten Metropolregio- \\ nen nämlich einen wichtigen Beitrag zu einer \\ nachhaltigen Regionalentwicklung leisten. \\ Von Jörg Knieling und Tobias Preising
}

D ie Einführung der Metropolregionen als Raumordnungskategorie hat seit 1995 nicht nur ungewöhnlich große Resonanz und Erwartungen seitens der betroffenen oder potenziell betroffenen Regionen gefunden, sondern auch in der Fachwelt wurde hiermit eine breite Diskussion in Gang gesetzt (Knieling 2009).

Eine mögliche Antwort auf die vielfältigen regionalen und auch internationalen Herausforderungen wie Globalisierung oder Strukturwandel der Wirtschaft wurde in Europa in der Entwicklung von mehreren starken Agglomerationsräumen gesehen. Diese Räume sollten nicht nur wichtige Standbeine der nationalen Entwicklung sein, sondern gleichzeitig eine Handlungsarena zur Entwicklung des europäischen Wirtschaftsraums werden (MKRO 1995; EU 1999). Dies ist seit Veröffentlichung der „Leitbilder und Handlungsstrategien für die Raumentwicklung in Deutschland“ verstärkt unter der neuen, programmatischen Bezeichnung Metropolregionen geschehen (MKRO 2006).

\section{Was sind Metropolregionen?}

Die Begriffsklärung erfordert eine Differenzierung zwischen analytischer und akteursbezogener Betrachtung sowie normativer und symbolischer Zielsetzung (ARL 2007):

- Analytisch sind Metropolregionen durch eine Ansammlung von metropolitanen Einrichtungen und einem Zusammentreffen von Metropolfunktionen gekennzeichnet (BBR 2007).

I Akteursbezogen stellen Metropolregionen einen Handlungsraum dar, dessen regionale Schlüsselakteure sich über gemeinsame regionale Ziele, Strategien und Projekte in den notwendigen Organisationsformen verständigen.

- Normativ sind Metropolregionen in Bezug auf die Raumentwicklung zukunftsgerichtete Leitvorstellungen, die sowohl das Potenzial von Innovation und Wirtschaftswachstum beschreiben und erhöhen wie auch eine nachhaltige Entwicklung sichern sollen.

- Symbolisch sollen Metropolregionen Assoziationen von Weltstadt, Urbanität und globaler Bedeutsamkeit hervorrufen und für die regionale Entwicklung nutzbar machen. Darüber hinaus sollen Metropolregionen zu einer Identifikation der Bevölkerung und ansässigen Unternehmen mit dem Wohn- beziehungsweise Standort beitragen.

\section{Selbstverständnis der Metropolregionen in Deutschland}

Während die Rahmen setzenden Vorgaben der Ministerkonferenz für Raumordnung nur eine sehr vage Umschreibung der Raumordnungskategorie Metropolregion liefern, haben die $\rightarrow$

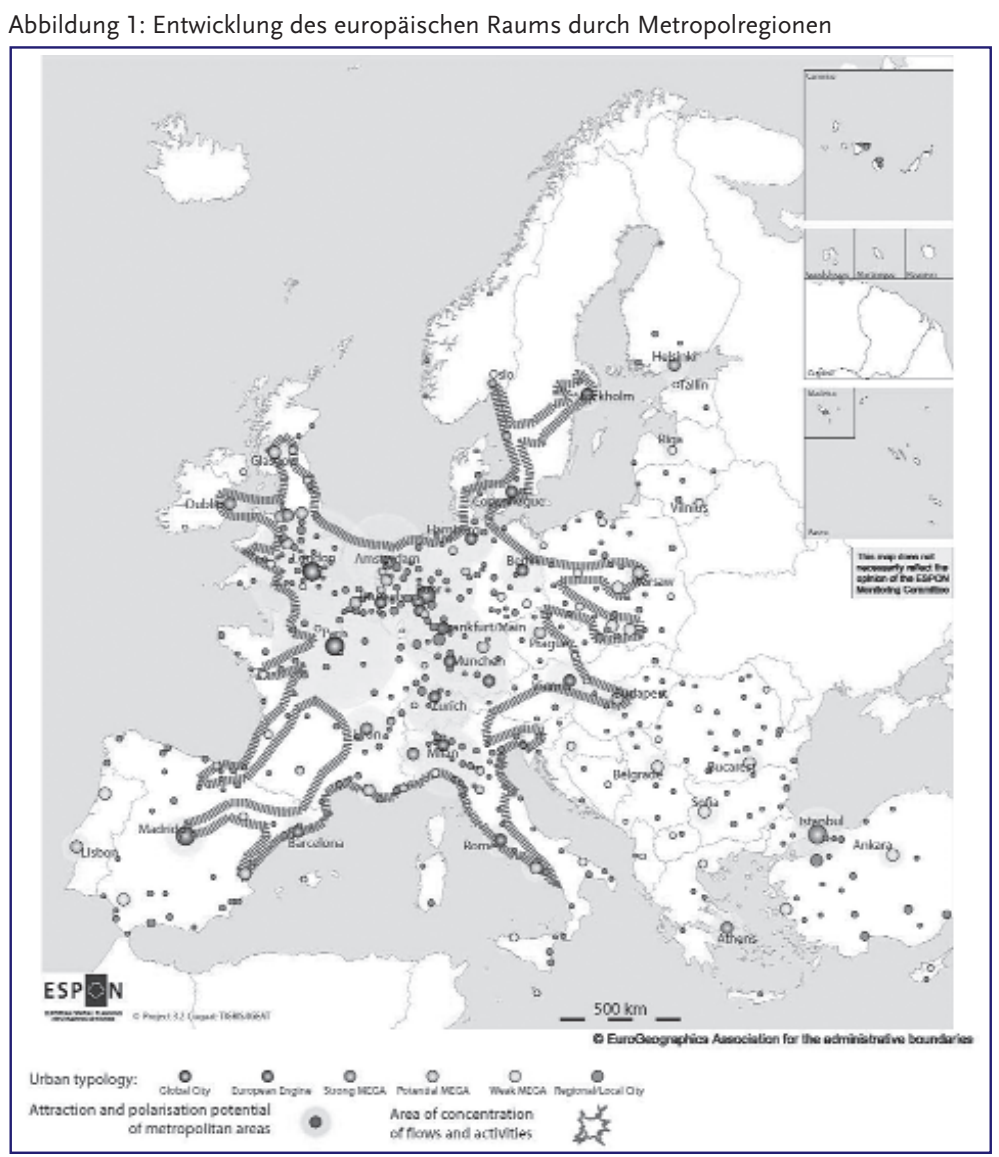

Quelle: ESPON 2007 
Metropolregionen über ihren Zusammenschluss im Initiativkreis Europäische Metropolregionen in Deutschland (IKM) selbst Bedingungen der Weiterentwicklung des Konzepts der Europäischen Metropolregion formuliert (MKRO 1995; MKRO 1997; IKM 2003).

Abgesehen von einer nötigen und abgestimmten individuellen Profilierung der Metropolregionen durch thematische Schwerpunktsetzungen sieht der IKM folgende Mindestaufgaben, die durch die Metropolregionen wahrgenommen werden sollen (IKM 2003):

I Strategische Planung in Form von Leitbildern und regionalen Entwicklungskonzepten,

- Regionalplanung und Koordinierung regional bedeutsamer Projekte und Maßnahmen,

- Siedlungssteuerung und Verkehrsplanung,

- Infrastrukturplanung in den Bereichen Kommunikation, Mobilität, Messe- und Kongresswesen, Sozialwesen, Erholung und Ökologie,

I Regionale Wirtschaftsförderung und Beschäftigungspolitik,

I Regionalentwicklung durch Regionalmanagement und -marketing sowie

- Wahrnehmung und Vertretung regionaler Interessen nach innen und außen.

Eine schriftliche Erhebung der Metropolregionen in 2008 hat im Gegensatz zu diesen engagierten Aufgabensetzungen ergeben, dass bisher eher weiche Themen die Arbeit dominieren

Abbildung 2: Aktuelle Handlungsfelder der Metropolregionen

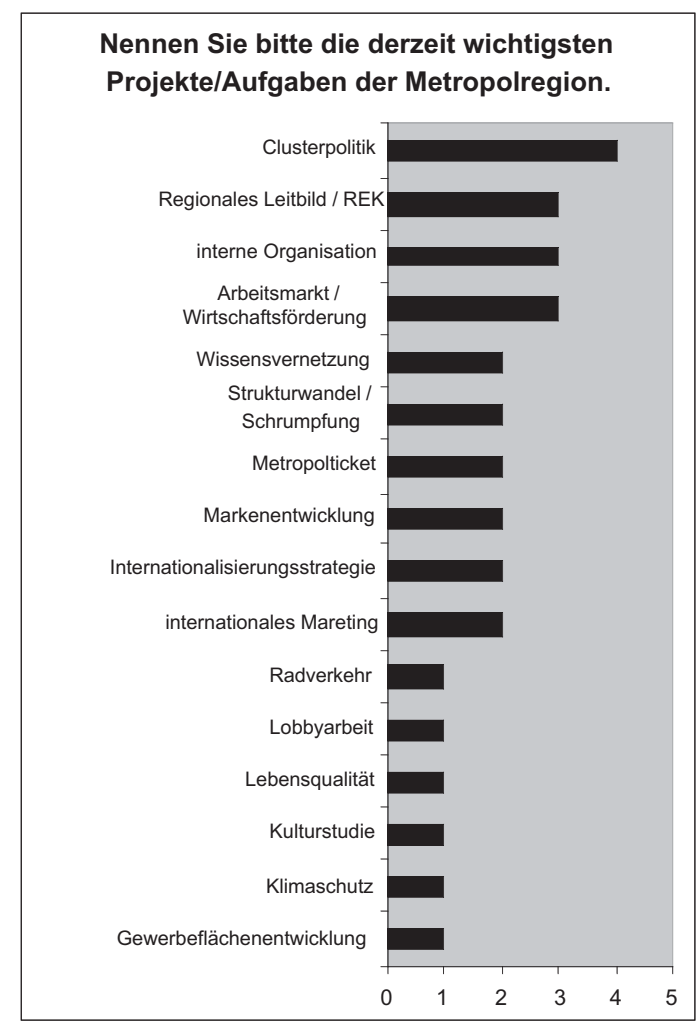

Quelle: eigene Darstellung und sich die Kooperation in den Regionen noch im Aufbau befindet. Als aktuelle Projekte werden neben Clusterpolitik und Wirtschaftsförderung demnach vorrangig der interne interkommunale Kooperationsaufbau, die Profilbildung nach außen und das klassische Standortmarketing auf internationaler Ebene genannt (siehe Abbildung 2).

Grundsätzlich lässt sich aber feststellen, dass das Konzept der Metropolregion eher positiv als Katalysator regionaler Prozesse gesehen wird. In Bezug auf eine räumliche Planung auf Ebene der Metropolregion werden zwar eher Chancen und Vorteile gesehen, aber es wird ebenso deutlich, dass dies erstens politisch gewollt sein muss und zweitens eine größere Kooperationsroutine erfordert, als sie derzeit in vielen Metropolregionen vorhanden ist. Dementsprechend ist auch die Bedeutung der räumlichen Planung bisher noch gering.

Dennoch besitzen bereits einige Metropolregionen ein integratives Leitbilddokument oder arbeiten aktuell daran und nur in drei der elf Metropolregion ist ein solches derzeit nicht vorhanden. In der abgefragten Charakterisierung des Konzepts der Metropolregion deutet sich auch an, dass die Entwicklung metropolregionaler Leitbilder eine Verschiebung von reiner Wirtschaftsförderung hin zu einer integrativen Regionalentwicklung mit sich bringt.

\section{Qualitäten von nachhaltigen Metropolregionen}

Handlungsfelder einer integrativen Regionalentwicklung mit dem Ziel einer nachhaltigen Metropolregion sollten sich nicht nur an den ökonomisch ausgerichteten Zielformulierungen der Lissabon-Strategie orientieren, sondern explizit weitere Dimensionen stadtregionaler Nachhaltigkeit erfassen:

-Wissensregion zur Stärkung der Ressourcen in Bildung und Wissensgesellschaft. Gerade im viel beschworenen Standortwettbewerb zählen zunehmend die wissensökonomischen Potenziale einer Region. Wesentliche Vorteile in der Bereitstellung von Wissen und kreativem Potenzial bieten Agglomerationen, die über eine vielfältige Bildungslandschaft verfügen.

Bereits heute konzentrieren sich kreative Entwicklungen in Szenevierteln und Subkulturen der Metropolen, die wie Berlin und Hamburg auch eine breite Palette klassischer Wissenseinrichtungen, beispielsweise Universitäten, Fachhochschulen und Forschungseinrichtungen vorweisen können.

I Zuwanderung und Integration für Innovation und Lebensqualität. Ein Problem der Sicherung des kreativen Potenzials der Gesellschaft ist die oft mangelnde Integrationsfähigkeit gegenüber Zuwanderung. Nach wie vor liegt beispielsweise die Quote der Bildungsabbrecher bei Schülern mit Migrationshintergrund deutlich über dem bundesdeutschen Durchschnitt. Hierdurch werden nicht nur persönliche Lebenskarrieren beschnitten, sondern auch Potenziale zur Weiterentwicklung der Metropolen zu inter- 
nationalen Orten in Subkulturen und gesellschaftliche Randlagen abgedrängt.

Hier wäre ein Paradigmenwechsel weg von der Forderung nach Assimilation von Zuwanderern hin zur Integration von neuen kulturellen Aspekten in eine weltoffene und globalisierte Gesellschaft von Nöten. Gerade im wissenschaftlichen Bereich stellt Zuwanderung eine wichtige Ressource an gut qualifizierten Talenten dar (siehe Abbildung 3).

I Konstruktiver Umgang mit den Risiken des globalen Klimawandels. Der globale Klimawandel ist eine der größten Herausforderungen in naher und mittelfristiger Zukunft. Die Agglomerationen sind die größte Emissionsquelle von Treibhausgasen und werden auch von den Folgen vermutlich am schwersten betroffen sein. Deshalb sollten sie sowohl in der Mitigation, der Vermeidung, als auch bei der Adaption, der Anpassung an die Folgen, eine zentrale Rolle spielen.

Gleichzeitig bietet der Klimawandel auch Handlungsfelder für neue Technologien. Bereits heute sind die deutsche Industrie und Forschung Technologieführer beispielsweise bei solaren Energiesystemen oder in der Windkraft.

- Nutzung der breiten, teilweise noch unerschlossenen Ressourcen der Zivilgesellschaft. Die Entwicklung zukunfts-

Abbildung 3: Herkunft und Bildungsabschluss von Zuwanderern

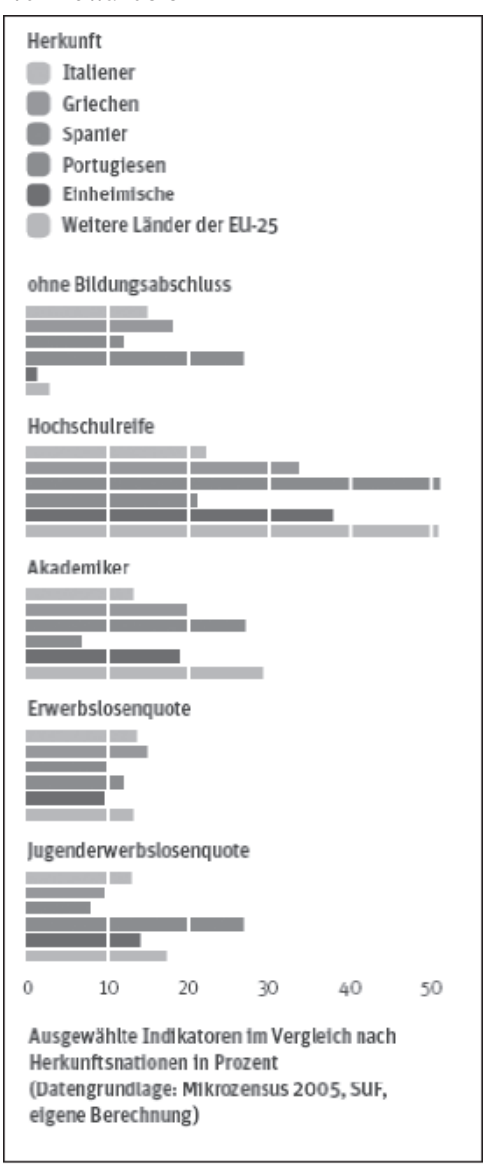

Quelle: Berlin-Institut 2009 weisender Strategien für Metropolregionen liegt $\mathrm{zu}$ meist in den Händen von institutionalisierten Akteuren, etwa Regionalverbänden. Zivilgesellschaftliche Akteure, die wichtige neue Impulse bereithalten oder auch nur als Multiplikatoren wirken könnten, finden noch zu wenig Eingang in die Entwicklung von strategischen Überlegungen.

I Soziale und territoriale Kohäsion. Die zunehmenden Disparitäten innerhalb der Bevölkerung stellen eine wichtige Herausforderung für die nachhaltige Entwicklung von Metropolregionen dar. Hier sind nicht nur die immer weiter auseinander driftenden Einkommensverhältnisse zwischen Reich und Arm, sondern auch der Zugang zu Bildung, zu Infrastrukturen, sozialen Netzwerken oder auch Freiräumen und kulturellen Einrichtungen gemeint.

\section{Perspektiven für nachhaltige Metropolregionen}

Die Lösung der aufgezeigten Herausforderungen ist eine komplexe Aufgabe und dies umso mehr in Metropolregionen, die durch eine Vielfalt an Akteuren und damit widerstreitenden Interessen gekennzeichnet sind.

Damit der Diskurs über Metropolregionen seine überwiegend einseitige Wachstums- und Wettbewerbsorientierung verliert, ist in jedem Fall eine thematisch umfassendere Betrachtung nötig. Dabei kann auf langjährige Erfahrungen und Konzepte integrativer Entwicklung der deutschen Großstadtregionen zurückgegriffen werden. Neue Handlungsfelder wie Klimawandel oder Wissensgesellschaft stellen aber Anforderungen, für die noch keine Routinen zur Verfügung stehen.

\section{Literatur}

ARL (Hrsg.): Metropolregionen - Innovation, Wettbewerb, Handlungsfähigkeit. Hannover 2007

Berlin-Institut (Hrsg.): Ungenutzte Potenziale. Zur Lage der Integration in Deutschland. Berlin 2009.

BBR: Bundesamt für Bauwesen und Raumordnung: Raumordnungsbericht 2005. Bonn 2007.

ESPON: Scenarios of the territorial future of Europe. o.O. 2007. Im Internet: http://www.espon.eu

EU: EUREK - Europäisches Raumentwicklungskonzept. Auf dem Wege zu einer räumlich ausgewogenen und nachhaltigen Entwicklung der Europäischen Union. Angenommen beim informellen Rat der für Raumordnung zuständigen Minister in Potsdam. Herausgegeben von der Europäischen Kommission. Brüssel 1999.

IKM: Initiativkreis Europäische Metropolregionen in Deutschland: Strategiepapier. Stand 31.03.2003. Berlin 2003.

MKRO: Ministerkonferenz für Raumordnung 1995: Raumordnungspolitischer Handlungsrahmen. Beschluss der MKRO vom 08.03.1995. Bonn 1995.

MKRO: Bedeutung der großen Metropolregionen Deutschlands für die Raumentwicklung in Deutschland und Europa. Beschluss der MKRO vom 3.6.1997. Bonn 1997.

MKRO: Leitbilder und Handlungsstrategien für die Raumentwicklung in Deutschland. Beschluss der MKRO vom 30.06.2006. Berlin 2006.

\section{AUTOREN + KONTAKT}

Dr. Jörg Knieling ist Professor und Dipl.-Ing. Tobias Preising Mitarbeiter im Fachgebiet Stadtplanung und Regionalentwicklung der HafenCity Universität Hamburg.

HafenCity Universität Hamburg, Fachgebiet Stadtplanung und Regionalentwicklung, Winterhuder Weg 31, 22085 Hamburg. Tel.: +49 40 42827-4515, Fax:+49 40 42878-4516,

E-Mail: joerg.knieling@hcu-hamburg.de; tobias.preising@hcu-hamburg.de
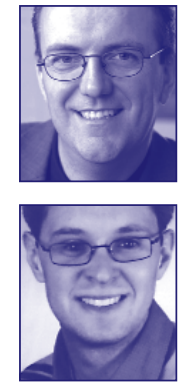
(c) 20I0 Authors; licensee IÖW and oekom verlag. This is an article distributed under the terms of the Creative Commons Attribution Non-Commercial No Derivates License (http://creativecommons.org/licenses/by-nc-nd/3.o/), which permits unrestricted use, distribution, and reproduction in any medium, provided the original work is properly cited. 